\title{
Quantitative study of stiffness in the knee joint
}

\author{
C. H. SUCH, A. UNSWORTH, V. WRIGHT, AND D. DOWSON \\ From the Bioengineering Group for the Study of Joints, Leeds University
}

\begin{abstract}
Such, C. H., Unsworth, A., Wright, V., and Dowson, D. (1975). Annals of the Rheumatic Diseases, 34, 286-291. Quantitative study of stiffiness in the knee joint. Data from a knee arthrograph, which imparts a passive sinusoidal motion to the joint and measures the resistive torque and displacement, have been shown to be reproducible. Tests carried out on joints (from 49 males, 21 females) showed that the energy loss per cycle of the joint increased with age for both sexes but the peak to peak torque (elastic stiffness) did not vary with age. Muscle bulk had a large influence on the stiffness (both dissipative and elastic) of the knee as did the size of the joint itself.

Women's knee joints exhibited lower values of dissipative energy loss and peak to peak torque than men's for all ages and sizes of joints.
\end{abstract}

Stiffness of joints is an important clinical feature in rheumatic disease. Patients find that stiffness imposes a great limitation in mobility of joints and is also associated with pain. Indeed, stiffness is a major diagnostic criterion in the detection of rheumatoid arthritis (Ropes, Bennett, Cobb, Jacox, and Jessar, 1959) and osteoarthrosis (Hollander, 1953). However; little objective measurement was produced to quantitate stiffness until the work of Wright and Johns (1960), who built a finger arthrograph based on the principle of a pendulum. This device imposed an angular motion of sinusoidal waveform on the metacarpophalangeal joint of the index finger. This particular waveform was used to calculate the accelerations of the finger, but an additional benefit was found to be that a sinusoidal waveform eliminated reflex muscle activity. Having imposed this motion on the joint, the resistance to this motion was measured by means of a strain gauged lever which allowed the torque to be plotted against angular displacement and also against angular velocity.

At the same time, Scott (1960) studied morning stiffness in rheumatoid arthritis using as a measure the deflection from the equilibrium position of the metacarpophalangeal joint of the index finger when loaded by a spring attached perpendicularly to the axis of the finger. He measured grip strength of normal subjects and patients with rheumatoid arthritis and showed in terms of these comparative tests the phenomenon of morning stiffness.

Wright and Johns (1961) modified the arthrograph and provided a driven system still imparting the $\vec{\varphi}$ sinusoidal motion to the metacarpophalangeal joif Long, Thomas, and Crochetiere (1964) studied theO rheology of hand control using a finger arthrograph based on that of Wright and Johns, showing the importance of the wrist position when carrying out these tests. Wright and Plunkett (1966) assessed $\mathbb{Q}^{\mathbb{D}}$ stiffness of the metacarpophalangeal joint of the in $-\overrightarrow{\vec{F}}$ dex finger by several methods including grip strength, $\frac{0}{3}$ the ability to tie knots, and the physical stiffness as measured by the arthrograph. Bäcklund and Tiselius (1967) and Barnett and Cobbold (1969) also studied joint stiffness by means of an arthrograph and $a_{0}^{\frac{2}{2}}$ pendulum apparatus, respectively.

At Leeds, a knee arthrograph was developed to study knee stiffness and articular gelling (Goddard,ô Dowson, Longfield and Wright, 1969a, b). In this communication is described how the knee arthro-o graph was used to provide more basic information on the stiffness of normal knees and factors which influence this.

\section{Knee arthrograph (Fig. 1)}

The subject was seated with his leg attached to the ${ }_{\sigma}$ drive arm of the apparatus such that no movement was permitted at the ankle when the tibia was rotated? relative to the femur. The thigh was always main- $-\infty$ tained horizontally. The foot rest at the lower end of the drive arm was adjustable so that the knee couldo be aligned with the axis of rotation of the drive arm. 


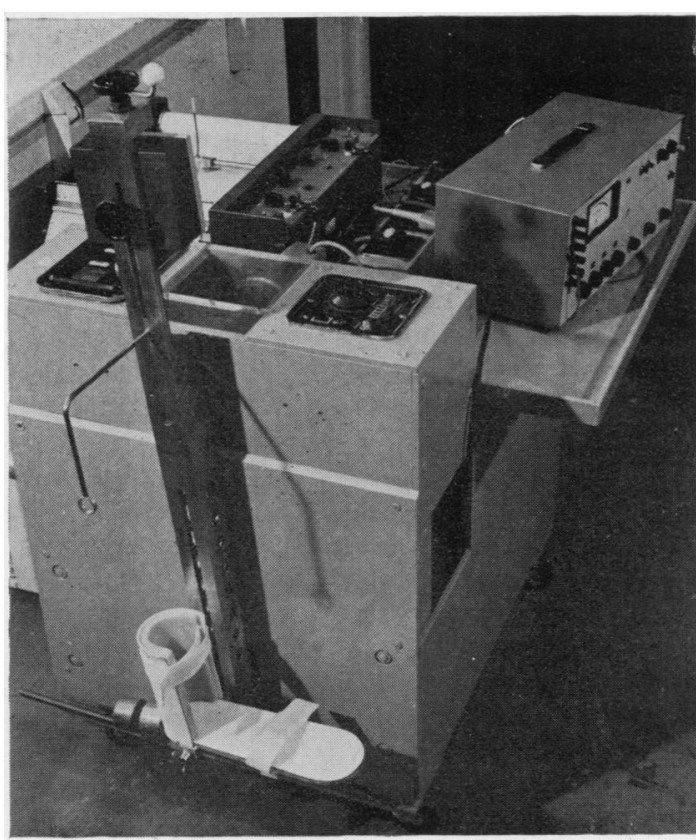

FIG. 1 The knee arthograph

No restraints were imposed on the tissues around the joint, and contact of the articular surfaces in the joint was ensured by the light loading imposed by the weight of the thigh plus the tension within the liagments.

The mechanism allowed for a variation in the amplitude of the oscillatory motion from zero to 2.5 radians and also for the degree of flexion of the tibia relative to the femur. This versatility allowed stiffness to be measured in the positions of full extension or full flexion, as well as intermediate amounts of flexion. The angular velocity of the motion applied to the leg was variable between zero and 0.45 radian per second. Stiffness was measured by a strain gauged plate. The effect of the weight of the leg itself was eliminated by means of a counter balance.

\section{Materials and method}

Seventy knees were tested from subjects without a clinical history of joint problems, 49 from males and 21 from females. The ages of the subjects ranged from 21 to 65 years.

The knee joint was aligned with the axis of rotation of the drive arm by means of adjustments to the foot rest. Due to the geometry of the knee, the axis of rotation varies as the joint is flexed and extended. For this reason, the concept of an 'effective' axis of rotation was introduced. The joint was aligned by means of an external 'sighting device' such that, for a particular amplitude of motion of the lower leg, no horizontal or vertical movement was detectable. In such a position the joint was considered to be rotating about its effective axis for that particular angle of flexion, and that this coincided with the axis of rotation of the drive arm of the arthrograph.
The tests were carried out at an angular velocity of 0.078 radian per second. This was slow enough to reduce the inertial effects of the leg and machine to negligible proportions. The subjects normally needed a few cycles of the arthrograph to become accustomed to the motion and hence relax. During this time any muscle contractions could be seen simply as large impulses on the normal resistive pattern recorded by the $x-y$ recorder. Once the subject had settled to the motion and uniform traces were being produced, five consecutive results were obtained and analysed. All values were then averaged from the five traces. Some subjects were unable to relax sufficiently for the traces to be repeatable and these results were discarded, and were not included in the numbers quoted at the beginning of the section.

On completion of the tests, the areas inside the loops were obtained by measurement using a planimeter. This represents the dissipated energy of the joint. The peak to peak torque was obtained by direct measurement from the traces.

\section{Results}

Fig. 2 shows a diagram representing the form of the torque-displacement traces. In this the various energy sources are marked (i.e. viscous and coulomb) as well as the elastic torque.

\section{REPRODUCIBILITY}

To test the reproducibility of the technique, the stiffness of the right knee of a 27-year-old man with no evidence of joint disease was measured. This test was repeated after a short period of rest during which the leg remained in position on the machine. Each test took about two minutes, and leaving a rest period of two minutes between each test, another four sets of results were taken. A similar test was also carried out on a 22-year-old man. The dissipated energy loss for the first subject varied by $2.9 \%$ over the tests (mean 5.447 , SD $0.08 \mathrm{Nm} /$ cycle) while the peak to peak torque varied by $9.7 \%$ (mean 4.31 , SD $0.33 \mathrm{Nm}$ ).

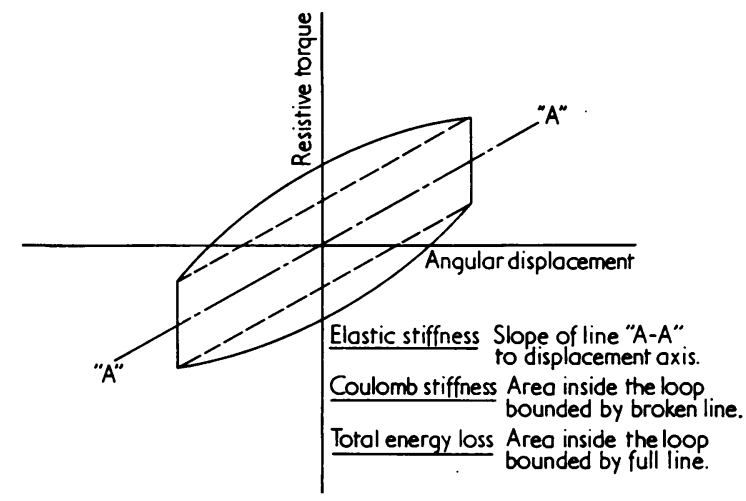

FIG. 2 Diagram of stiffness trace 


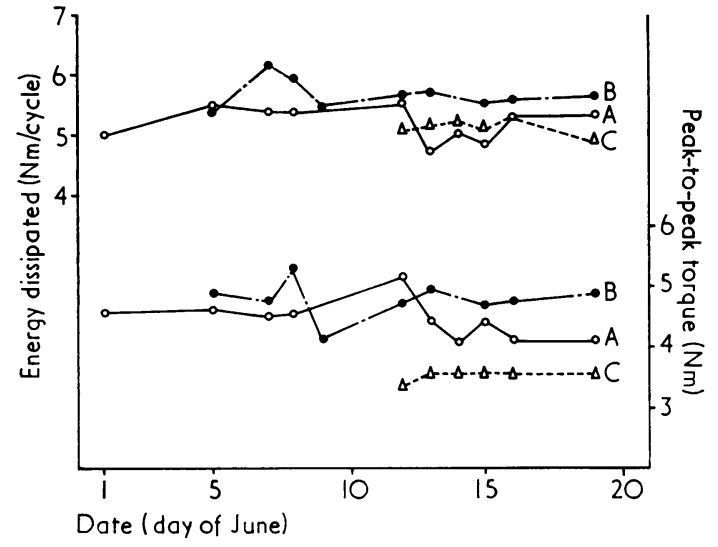

FIG. 3 Day-to-day variation of knee stiffness in 3 males. $A=27$ years; $B=25$ years; $C=38$ years

The day-to-day variation of the stiffness of the knees of three men was investigated over 3 weeks (Fig. 3). The variations in dissipative stiffness was $2 \cdot 6-7 \cdot 2 \%$ and elastic stiffness $2 \cdot 4-7 \cdot 2 \%$. Subject $A$ had greatest coefficient of variation due to the fact that he undertook a 30-mile hike across rough moorland during the trial and complained of stiffness in his knees afterwards! Fig. 3 shows this increase in elastic torque on June 12, the walk having taken place on June 10.

\section{FACTORS WHICH INFLUENCE JOINT STIFFNESS}

Age

Dissipated energy increased with advancing age (Fig. 4). The increase in dissipated energy from the

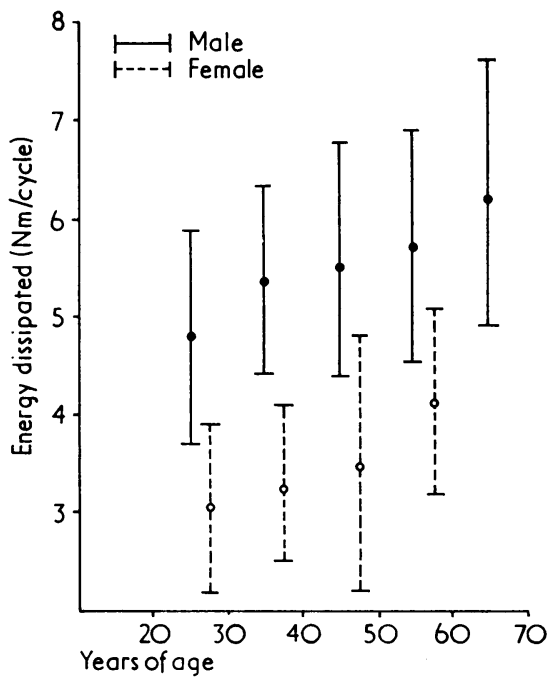

FIG. 4 Variation in dissipated energy with age 2nd to the 6th decades was $23.2 \%$ in men and $33 \%$ in women. Some in the 6 th decade experienced the same stiffness as some in the 2 nd decade, but the increase $\frac{}{n}$. in stiffness between the two decades was significant: $(\mathrm{P}<0.05)$. Adjacent decades did not prove to be significantly different from one another. Peak to peak torque was unchanged throughout the age range $\frac{\overline{0}}{-}$ (Fig. 5). This was contrasted with the earlier work of $\overline{\bar{c}}$ Wright, Dowson, and Longfield (1969) on the meta- $-\widehat{\Phi}$ carpophalangeal joint.

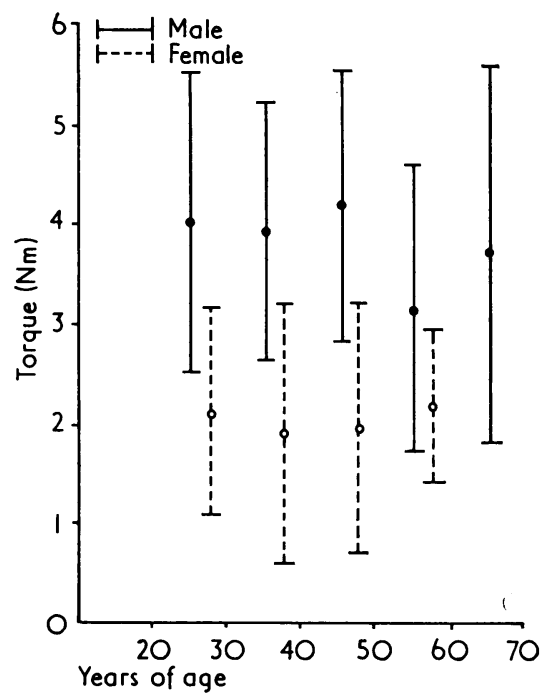

FIG. 5 Variation in elastic stiffness with age

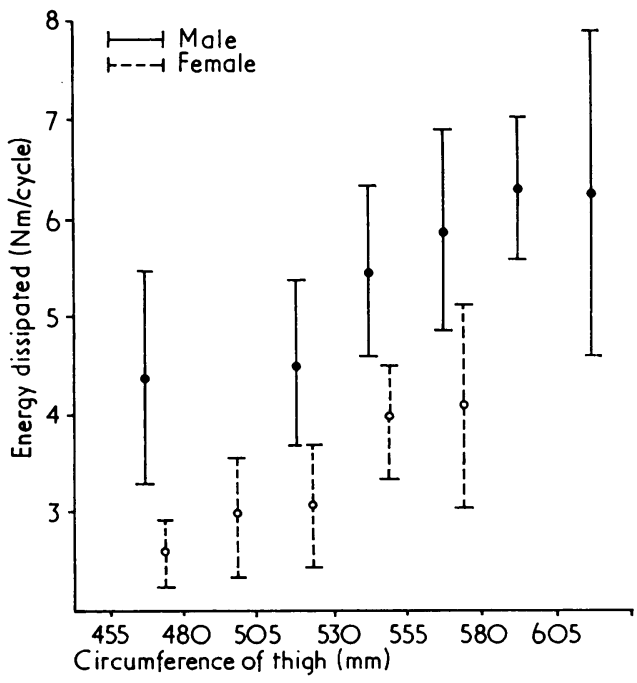

FIG. 6 Variation in dissipated energy with circumference of thigh 


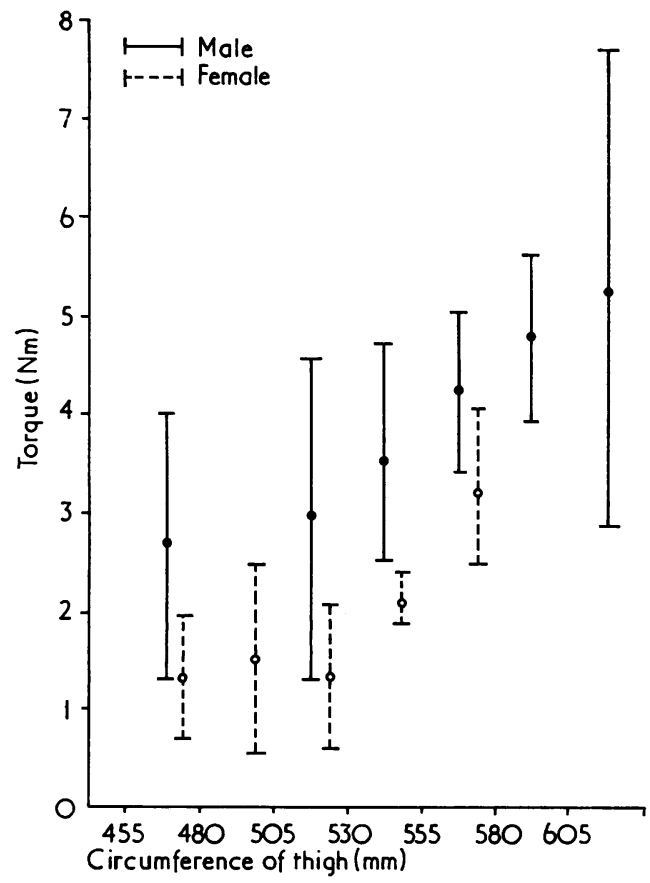

FIG. 7 Variation in elastic stiffness with circumference of thigh

Sex

Women exhibited lower dissipated energy than men and also less peak to peak torque. The dissipated energy cycle was on average $55.9 \%$ greater for men than women, while the peak to peak torque was $84 \%$ greater in men than women.

\section{Size of thigh}

Using the knee arthrograph, Adams (1973) studied the effects of exercise of professional soccer players on the stiffness of their knees. He noted a relationship between the size of the thigh and stiffness. In this study, therefore, the circumference of the thigh was measured half-way between the anterior iliac spine and the proximal edge of the patella, and the knee stiffness was plotted against this variable (Figs 6 and 7).

Both dissipated energy and peak to peak torque increased with muscle size, the energy loss increasing by $30 \%$ in males and $36 \%$ in females as the thigh circumference varied from $470 \mathrm{~mm}$ to $622 \mathrm{~mm}$. Peak to peak torque increased by $48.8 \%$ in males and 58.5 $\%$ in females. These increases were significant $(\mathrm{P}<0.05)$.

\section{Size of knee}

As a rough guide to the amount of structures surrounding the knee, such as tendons and ligaments, the circumference of the leg was taken at a point $50 \mathrm{~mm}$ above the proximal edge of the patella. This was then plotted against knee stiffness (Figs 8 and 9).

Both dissipated energy and peak to peak torque increased with knee circumference such that the energy loss per cycle increased by $41.5 \%$ in males,

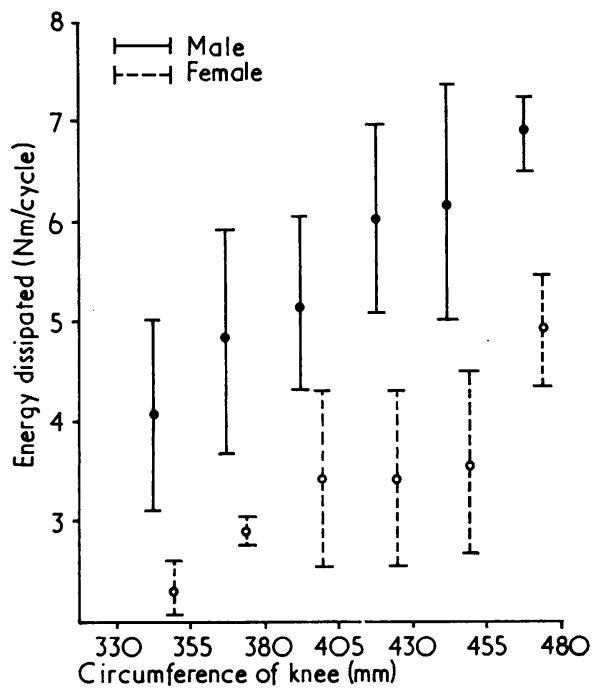

FIG. 8 Variation in dissipated energy with circumference of knee

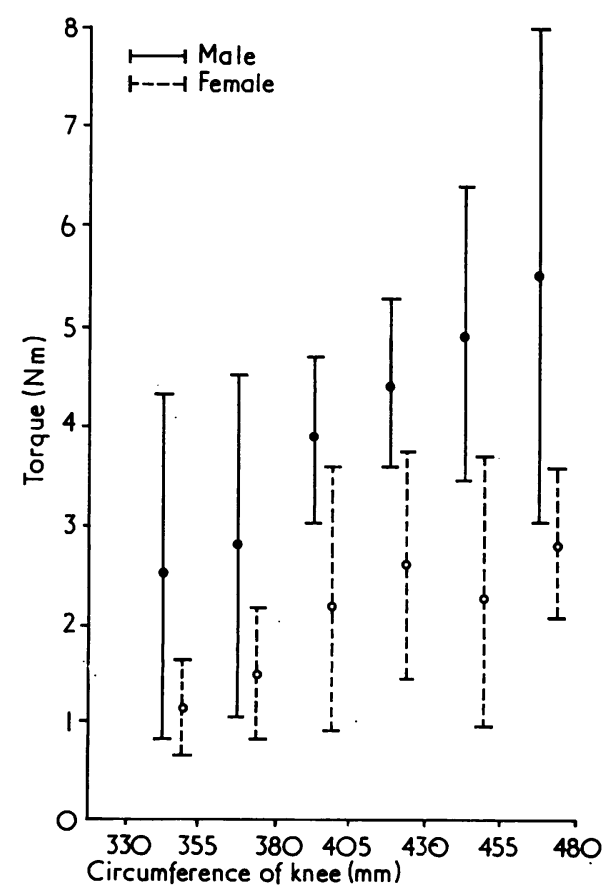

FIG. 9 Variation in elastic stiffness with circumference of knee 
$53.2 \%$ in females, and the elastic torque increased by $55.5 \%$ in males and $58.6 \%$ in females as the knee circumference increased from 340 to $470 \mathrm{~mm}$.

\section{Discussion}

A clear relationship between age and knee stiffness has emerged from this work and the indications are that while dissipated energy increases with age, elastic (peak to peak) torque is unchanged. Dissipated energy might well be expected to increase as age increases since some factors which influence energy loss are known to alter with age. For instance, Walker, Dowson, Longfield, and Wright (1968) showed that surface roughness of articular cartilage increases with age and this could have a considerable effect on the frictional properties of cartilage. Also synovial fluid becomes less viscous with advancing years (Jebens and Monk-Jones, 1959), therefore being less efficient as a lubricant. Cartilage itself becomes less elastic in old age (Barnett and Stockwell, 1966), and if some form of elastohydrodynamic lubrication process is important (Dowson, 1966), then this will have a significant influence on the resultant frictional forces. It seems logical, therefore, that if the changes observed by other workers are real, then the resultant effect will be to increase the dissipative energy of the joints as the joint becomes older.

The lack of change in the elastic torque with age is less satisfactorily explained. Collagen becomes more rigid with age (Wright and Johns, 1960) and as a result one might expect the elastic torque to increase. However, structures such as ligaments and tendons often become lax with age. This means that the structures have become permanently stretched and so, on first moving the joint, low resistance is encountered until all the 'slack' is taken up. After this the stiffness should be great. Overall, the two effects could neutralize one another. The result would therefore be little change in elastic stiffness with age.

The effect of muscle size is to be expected in that a greater mass of tissue of similar elastic modulus will produce a greater elastic resistance when movement takes place. Similarly, more viscous loss will occur if a larger number of fibres are sliding over one another. These effects are in agreement with the work of Wright and Johns (1960) on a cat's wrist joint in which passive extension of the muscles accounted for $40 \%$ of the total stiffness.

The size of the knee was important for similar reasons. Tendons with large cross-sectional areas would exhibit stiffer properties than smaller tendons, and in addition create higher viscous losses as they slide within sheaths and over other tissues. This again agrees with the work of Wright and Johns (1960) on the cat wrist joint which showed that $10 \%$ of the stiffness was attributable to the tendons and $47 \%$ to the capsule itself.

There appears to be a substantial difference in stiffness between male and female joints. Males are much stiffer than females even when age, size of thigh, and size of knee is taken into account. It interesting to note that while this work shows that. females are less stiff than males, Macrae and Wright (1969) and Moll and Wright (1971) have shown that females have less back mobility and a smaller chest expansion than males.

We would like to thank the Arthritis and Rheumatism Council for their financial support throughout this project.

\section{References}

ADAMS, D. I. (1973) M.D. Thesis, Leeds. (Osteoarthrosis of the knee joint in sportsmen)

BÄCKLund, L., AND Tiselius, P. (1967) Acta rheum. scand., 13, 275 (Objective measurement of joint stiffness in rheumatoid arthritis)

Barnett, C. H., AND CoBbold, A. F. (1969) Ann. rheum. Dis., 28, 6 (Muscle tension and joint mobility)

-, AND STOCKWELL, R. A. (1966) 'The aetiology of osteoarthrosis', in 'Modern Trends in Rheumatology', ed. A. G. S. Hill, p. 247. Butterworths, London

Dowson, D. (1966) Proc. Instn. Mech. engrs., 181, 45 (Modes of lubrication in human joints)

Goddard, R., Dowson, D., Longfield, M. D., AND Wright, V. (1969a) Rheologica Acta, 8, 229 (The measurement of stiffness in human joints)

$\ldots,-\ldots, \ldots,(1969 \mathrm{~b})$ 'A study of articular gelling', in 'Lubrication and Wear in Human Joints', ed. V. Wright, p. 134. Sector, London

Hollander, J. L. (1953) In 'Arthritis', 5th ed., p. 25. Lea and Febiger, Philadelphia

Jebens, E. H., AND MONK-JoNes, M. E. (1959) J. Bone Jt. Surg., 41B, 388 (On the viscosity and pH of synovial fluid and the $\mathrm{pH}$ of blood)

LONG, C., ThOMAS, D., AND CROCHetIERe, W. J. (1964) 'Viscoelastic factors in hand control', in 'Proceedings of the Fourth International Congress of Physical Medicine, Paris, 1964', p. 440. Excerpta Medica, Amsterdam

MACRAE, I. F., AND Wright, V. (1969) Ann. rheum. Dis., 28, 329 (Measurement of lumbar spine motion in population studies)

Moll, J. M. H., AND Wright, V. (1971) Ann. rheum. Dis., 30, 381 (Normal range of spinal mobility; an objective clinical study) 
Ropes, M. W., Bennett, G. A., CobB, S., Jacox, R., AND Jessar, R. A. (1959) Arthr. and Rheum., 2, 16 (1958 revision of diagnostic criteria for rheumatoid arthritis)

Scotr, J. T. (1960) Ann. rheum. Dis., 19, 361 (Morning stiffness in rheumatoid arthritis)

Walker, P. S., Dowson, D., Longfield, M.D. and Wright, V. (1968) Ann. rheum. Dis., 27, 512 (Boosted lubrication in synovial joints by fluid entrapment and enrichment)

Wright, V., Dowson, D., AND LoNGFIELD, M. D. (1969) Bio-med. Eng., 4, 8, 160, 517 (Human joint lubrication)

Wright, V., AND Johns, R. J. (1960) Bull.Johns Hopk. Hosp., 106, 215 (Physical factors concerned with the stiffness of normal and diseased joints)

Wright, V., AND Johns, R. J. (1961) Ann. rheum. Dis., 20, 36 (Quantitative and qualitative analysis of joint stiffness in normal subjects and in patients with connective tissue diseases)

Wright, V., AND Plunkett, T. G. (1966) Ann. phys. Med., 8, 280 (Scientific assessment of the results of physical treatment-measurement of stiffness) 